\title{
Ischemic stroke following intracranial hemorrhage from moyamoya disease
}

\author{
Satoru Takeuchi • Yoshio Takasato
}

Received: 13 February 2011 /Accepted: 17 February 2011 /Published online: 12 March 2011

(C) Springer-Verlag 2011

Sir,

We read with great interest the article entitled "Ischaemic stroke after acute intracranial hemorrhage in patients with moyamoya disease: six new cases and a short literature review" by Kim et al. [4]. The authors reported six cases of ischemic stroke after intracranial hemorrhage (ICH) from moyamoya disease (MMD), and reviewed the literature. Their review showed that the vast majority of patients (94\%) had intraventricular hemorrhages (IVH) without subarachnoid hemorrhage, and revealed that most cerebral infarcts (CI) after ICH from MMD were multiple, large, and cortically involved. The authors suggested that thromboembolism or vasospasm might play a role in the pathogenesis of CI after ICH from MMD. We completely agree with the authors' findings and wish to provide further comment.

We also believe that vasospasm is one of the contributing factors to $\mathrm{CI}$ after ICH from MMD. Preventive measures to reduce the risks of $\mathrm{CI}$ after ICH from MMD are important, because the optimal treatment strategy remains unclear. Su et al. [6] reported a case of CI after IVH from MMD, and suggested that early perfusion computed tomography (CT) scanning was a powerful tool for identifying patients at high risk of CI. Additionally, Ko et al. [5] reported that IVH volume was a prognostic factor for cerebral vasospasm. We suggest that perfusion CT scanning may be especially useful for predicting cerebral vasospasm in patients with massive IVH from MMD. There is no evidence for the optimal treatment for

S. Takeuchi $(\bowtie)$

Department of Neurosurgery, National Defense Medical College,

3-2 Namiki,

Tokorozawa, Saitama 359-8513, Japan

e-mail: s.takeuchi@room.ocn.ne.jp

Y. Takasato

Department of Neurosurgery, National Hospital Organization

Disaster Medical Center,

Tokyo, Japan
IVH from MMD; however, dehydration and hypotension should be avoided to prevent CI, regardless of the mechanisms involved [2]. Some authors have reported a relationship between increased intracranial pressure and cerebral vasospasm [3], and surgical interventions, such as ventricular drainage and/or endoscopic removal of the hematoma, may thus be indicated if increased intracranial pressure is suspected. Nimodipine or nicardipine may also be effective for preventing or treating cerebral vasospasm after ICH from MMD [1]. Further investigations into the optimal methods of predicting, preventing, and treating CI after ICH from MMD are required.

Conflicts of interest None.

\section{References}

1. Deshaies EM, Boulos AS, Drazin D, Popp AJ (2009) Evidencebased pharmacotherapy for cerebral vasospasm. Neurol Res 31:615-620

2. Iwama T, Kotani Y, Yamakawa H, Nagata I, Hashimoto N, Sakai N (2001) Cerebral ischemic complications following intracranial bleeding in patients with moyamoya disease-three case reports. Neurol Med Chir (Tokyo) 41:450-453

3. Karnchanapandh K (2008) Effect of increased intracranial pressure on cerebral vasospasm in SAH. Acta Neurochir Suppl 102:307-310

4. Kim DS, Jang DK, Huh PW, Yoo DS, Han YM, Huh CW (2011) Ischaemic stroke after acute intracranial haemorrhage in patients with moyamoya disease: six new cases and a short literature review. Acta Neurochir. doi:10.1007/s00701-010-0926-9

5. Ko SB, Choi HA, Carpenter AM (2011) Quantitative analysis of hemorrhage volume for predicting delayed cerebral ischemia after subarachnoid hemorrhage. Stroke 42:669-674

6. Su IC, Yang CC, Wang WH, Lee JE, Tu YK, Wang KC (2008) Acute cerebral ischemia following intraventricular hemorrhage in moyamoya disease: early perfusion computed tomography findings. J Neurosurg 109:1049-1051 